EGU21-5064

https://doi.org/10.5194/egusphere-egu21-5064

EGU General Assembly 2021

(c) Author(s) 2021. This work is distributed under

the Creative Commons Attribution 4.0 License.

\title{
Numerical simulation of coupled processes in a single fracture employing a continuum approach
}

\author{
Iman Vaezi ${ }^{1,2}$, Víctor Vilarrasa ${ }^{1,2,3}$, Francesco Parisio ${ }^{4}$, and Keita Yoshioka ${ }^{5}$ \\ ${ }^{1}$ Institute of Environmental Assessment and Water Research, Spanish National Research Council, Barcelona, Spain \\ ${ }^{2}$ Associated Unit: Hydrogeology Group UPC-CSIC, Barcelona, Spain \\ ${ }^{3}$ Mediterranean Institute for Advanced Studies, Spanish National Research Council, Esporles, Spain \\ ${ }^{4}$ Chair of Soil Mechanics and Foundation Engineering, Freiberg University of Mining and Technology, Germany \\ ${ }^{5}$ Department of Environmental Informatics, Helmholtz Centre for Environmental Research - UFZ, Germany
}

Fractures control fluid flow and the coupled geomechanical response of geological media in many geo-engineering applications. For instance, fractures dominate fluid flow and deformation in enhanced geothermal systems, underground radioactive waste repositories, and $\mathrm{CO}_{2}$ storage. Coupled thermo-hydro-mechanical processes in rock masses are a result of perturbations in the pore pressure, as in fluid injection and/or production, and/or temperature, as in cold fluid injection and disposal of radioactive waste. For example, fractures open as a result of pore pressure increase, which simultaneously increases permeability and reduces overpressure.

Geo-engineering and geo-energy applications involve a large portion of rock masses that include several fractures. Numerical computations of coupled processes occurring in rock masses while considering a large number of fractures pose several challenges. In this study, we firstly focus on a simple problem to fully understand the hydro-mechanical behavior of a single fracture subjected to a constant injection flow rate. We use the FEM software CODE_BRIGHT, which solves the thermohydro-mechanical governing equations in a fully coupled way. Since standard FEM can solve equations in continuum media, we investigate the behavior of a single fracture by analyzing the hydro-mechanical parameters that control the fracture response in a continuum fashion. However, simulating fractures with the real aperture is not simply feasible, hence, we search the equivalent properties of thicker fractures that are more feasible to be discretized in large-scale models with several fractures.

As the pore pressure increases inside a fracture, the fracture aperture increases and enhances its transmissivity. The embedded model uses variable permeability as a function of the cubic law. The simulation results show that a continuum approach can represent a fracture with a relatively large thickness (in the $\mathrm{cm}$ order) instead of the real aperture dimension (in the order of the micron). 